\title{
Analysis of programmed cell death in mouse fetal oocytes
}

\author{
A M Lobascio, F G Klinger, M L Scaldaferri, D Farini and M De Felici \\ Section of Histology and Embryology, Department of Public Health and Cell Biology, University of Rome Tor Vergata, \\ Via Montpellier 1, 00133 Rome, Italy
}

Correspondence should be addressed to M De Felici; Email: defelici@uniroma2.it

A M Lobascio and F G Klinger contributed equally to this work

\begin{abstract}
We report a short-term culture system that allows to define novel characteristic of programmed cell death (PCD) in fetal oocytes and to underscore new aspects of this process. Mouse fetal oocytes cultured in conditions allowing meiotic prophase I progression underwent apoptotic degeneration waves as revealed by TUNEL staining. TEM observations revealed recurrent atypical apoptotic morphologies characterized by the absence of chromatin margination and nuclear fragmentation; oocytes with autophagic and necrotic features were also observed. Further characterization of oocyte death evidenced DNA ladder, Annexin V binding, PARP cleavage, and usually caspase activation (namely caspase-2). In the aim to modulate the oocyte death process, we found that the addition to the culture medium of the pan-caspase inhibitors Z-VAD or caspase-2-specific inhibitor Z-VDVAD resulted in a partial and transient prevention of this process. Oocyte death was significantly reduced by the antioxidant agent NAC and partly prevented by KL and IGF-I growth factors. Finally, oocyte apoptosis was reduced by calpain inhibitor I and increased by rapamycin after prolonged culture.

These results support the notion that fetal oocytes undergo degeneration mostly by apoptosis. This process is, however, often morphologically atypical and encompasses other forms of cell death including caspase-independent apoptosis and autophagia. The observation that oocyte death occurs mainly at certain stages of meiosis and can only be attenuated by typical anti-apoptotic treatments favors the notion that it is controlled at least in part by stage-specific oocyte-autonomous meiotic checkpoints and when activated is little amenable to inhibition being the oocyte able to switch back and forth among different death pathways.
\end{abstract}

Reproduction (2007) 134 241-252

\section{Introduction}

In mammals, oogenesis begins with the formation of primordial germ cells and encompasses a series of cellular differentiation events, from primordial germ cells to oogonia, from oogonia to oocytes, and from oocytes to eggs. Extensive degeneration of germ cells has been described during embryonic, fetal, and early postnatal stages of oogenesis before follicle formation. In the mouse embryo, early morphological studies have shown that cell death may occur in primordial germ cells or oogonia (12-13 days post coitum, dpc), but mainly in oocytes at the zygotene/pachytene stage of meiotic prophase I (MPI; from 16.5 dpc through birth; Bakken \& McClanahan 1978). Following such episodes, the number of germ cells decreases from $\sim 20000$ at $13.5 \mathrm{dpc}$ to about 6000-10 000 after 6 days, at birth (Burgoyne \& Baker 1981, Tam \& Snow 1981). More recently, McClellan et al. (2003) performed a careful study of the number of oocytes throughout MPI in the embryonic mouse ovaries and reached the conclusion that the decrease of oocyte population during this period (about $65 \%$ loss) is a continuous process without apparent peaks of degeneration. If or not oocyte degeneration is a continuous or a stage-dependent process, it shows characteristics of programmed cell death (PCD). That is cell death which occurs in predictable places (fetal ovary) and at predictable times (mid-late gestation) and results in rapid elimination of considerable numbers of cells (oocytes) without any inflammatory reaction. Three types of PCD have been described. Type I is apoptotic cell death, which includes the morphological changes of cell shrinkage, membrane blebbing, and extensive chromatin condensation followed by nucleus fragmentation. Type II is autophagic cell death, characterized by the formation of autophagic vacuoles in the cytoplasm of dying cells. Type III is necrotic cell death, characterized by a rapid loss of plasma membrane integrity and spillage of the intracellular contents.

According to several reports, fetal oocyte PCD takes place in the form of apoptosis (for references, see De Felici et al. 2005). The process of oocyte death remains, however, incompletely characterized and alternative forms of PCD for embryonic germ cells remain possible 
(Wartenberg et al. 2001). Furthermore, the causes and the molecular mechanisms underlying oocyte PCD are little understood (for reviews, see Morita \& Tilly 1999, Tilly 2001, De Felici et al. 2005). Apoptosis may occur via a death receptor-dependent (extrinsic) or receptorindependent (intrinsic) pathway. The extrinsic pathway is activated by the binding of ligands (Fas ligand and tumour necrosis factor) to death receptors on the cell membrane. There is no evidence that such a pathway can operate in fetal oocytes (for a review, see De Felici et al. 2005). The intrinsic pathway is triggered by various extracellular and intracellular stresses, such as growth factor withdrawal, hypoxia, and DNA damage. On these bases, two main hypotheses have been advanced to explain germ cell death in the embryo. Germ cells like most other cell types could depend on growth factors for survival, and a limited supply of growth factors might determine oocyte apoptosis. In fact, the primordial germ cells, the precursors of both oocytes and spermatogonia, appear to undergo apoptosis in the absence of certain growth factors such as kit ligand $(\mathrm{KL}$, also known as stem cell factor) and leukemia inhibitory factor (LIF; for a review, see De Felici 2000). Evidence is increasing that fetal oocytes also need specific growth factors to avoid apoptosis. Growth factors reported to exert direct and/or indirect anti-apoptotic effect on the fetal oocytes include besides KL and LIF also insulin growth factor-I (IGF-I), interleukin $\alpha / \beta$, neurotrophin $4 / 5$, and brainderived neurotrophic factors (for reviews, see Morita \& Tilly 1999, Tilly 2001, De Felici et al. 2005). In the fetal ovary, growth factor dependence for survival could represent a way to limit the number of oocytes supported by somatic cells during the formation of follicles. In addition or alternatively, oocytes with defects in chromosome crossover, a process requiring critical events such as chromosome pairing, recombination, DNA repair, and synapses, might be eliminated by apoptosis to assure oocyte quality control. In the mouse, genetic mutations resulting in crossover defects during prophase I, which cause fetal oocyte depletion, have been identified (i.e. Atm, Barlow et al. 1998, Msh4 and Msh5, de Vries et al. 1999, Edelmann et al. 1999, Kneitz et al. 2000; Dmc1, Yoshida et al. 1998). Moreover, female mice that lack a second $X$ chromosome (X0) show oogenesis failure presumably due to failed chromosome pairing, which leads to increased oocyte degeneration during the development of the fetal ovaries (Burgoyne \& Baker 1985). A similar situation occurs in the Turner (X0) syndrome in humans, which is associated with massive oocyte apoptosis in the fetal ovary (Modi et al. 2003).

Though inadequate supply of growth factors and defects in the crossover are likely causes of fetal oocyte apoptosis, only little is known about the molecular pathways which in oocytes link such deficiencies to PCD.
A diverse spectrum of pro- and anti-apoptotic susceptibility genes including members of the $\mathrm{BCl}-2$ (Bax, BCl-x) and casp (caspase-2) gene families have been reported to be expressed in germ cells of the ovary and seem to be involved in controlling apoptotic pathways in oocytes (for a review, see Tilly 2001). While mice lacking Bax did not show alteration in the number of oocytes at birth (Perez et al. 1999), BCl-x- or $B C l$-2-deficient mice show a marked reduction of the postnatal oocyte pool (Ratts et al. 1995, Rucker et al. 2000). Moreover, evidence exists that increased levels of ceramide (Bergeron et al. 1998) and activation of the aromatic hydrocarbon receptor (Matikainen et al. 2001) may trigger apoptosis in fetal mouse oocyte.

All these data indicate that multiple mechanisms and complex pathway relationships may be responsible for the activation of PCD in fetal oocytes and further studies are needed to achieve a better characterization of this process. An impediment in such investigation has been the lack of an appropriate system for experimental analyses.

In the present study, we employed a simple culture system with the aim to analyze various aspects of PCD in mouse fetal oocytes and to provide a reliable experimental model in which this crucial process of early oogenesis can be studied at molecular level.

\section{Materials and Methods \\ Oocyte isolation and culture}

Oocytes were isolated from the ovaries of CD-1 mouse embryos by EDTA-stab method as described in De Felici \& McLaren (1982). This method allows to isolate monodispersed ovarian cell populations containing about $70-80 \%$ oocytes as revealed by morphological evaluation (contaminating somatic cells are easily identified on the basis of their small size and interphase nucleus) and positive staining with the oocyte-specific anti-germ cell nuclear antigen (GCNA)- 1 antibodies (Enders \& May 1994). Cells (about $2 \times 10^{4}$ oocytes $/ \mathrm{ml}$ ) were cultured in a Falcon tube (cat. No. 2005) at $37^{\circ} \mathrm{C}$ in $5 \% \mathrm{CO}_{2}$ for the indicated times, in $0.5 \mathrm{ml}$ modified MEM supplemented with $5 \%$ horse serum (HS), and $2.5 \%$ heat-inactivated fetal calf serum (FCS; Flow). In the experiments in which serum-free medium was used, $10 \mathrm{mg} / \mathrm{ml} \mathrm{BSA} \mathrm{(Fraction} \mathrm{V;} \mathrm{Sigma)} \mathrm{was} \mathrm{added} \mathrm{to} \mathrm{the}$ culture medium. KL (mouse recombinant) and IGF-I (human recombinant) were purchased from R\&D System (Space Import Export, Milan, Italy), LIF (mouse ESGRO) from Life Technology (Milan, Italy), and the caspase inhibitors Z-VAD-FMK and Z-DVAD-FMK from Alexis (Vinci, Italy). Calpain inhibitor I (N-Ac-leu-leu-norleucinal, ALLN) was obtained from Calbiochem (La Jolla, CA, USA) and rapamycin from Alexis.

All animal experimentation described in the present study were conducted in accord with the accepted standard of humane animal care. 


\section{GCNA-1 and SCP-3 staining}

For GCNA-1 labeling, $20 \mu \mathrm{l}$ ovarian cell suspension were spotted onto L-polylysine-coated slides and fixed with $4 \%$ paraformaldehyde for $10 \mathrm{~min}$, washed twice each for 10 min in PBS containing 0.5\% BSA. A 15-min block in PBS containing 0.3\% BSA, 0.05\% Triton X-100, and $1 \%$ goat serum was performed. Samples were then incubated overnight with 1:2 anti-GCNA-1 antibody, kindly provided by Dr G Enders (University of Kansas, Kansas, KS, USA; Enders \& May 1994). The next day the slides were washed in PBS plus $0.5 \%$ BSA and incubated for $45 \mathrm{~min}$ with a goat anti-rat IgM fluorescein isothiocyanate (FITC)-conjugated antibody (AlexaFluor; Invitrogen).

The meiotic prophase stages were determined by characteristic patterns of synaptonemal complex protein 3 (SCP-3) immunolabeling in spread cells, as described in Mahadevaiah et al. (2001). Briefly, cells were fixed with $2 \%$ paraformaldehyde containing $0.05 \%$ Triton X-100 and $0.02 \%$ SDS directly onto the slide. Samples were then washed and incubated in PBS-T $(0.15 \%$ BSA, $0.1 \%$ Tween 20 in PBS) for $1 \mathrm{~h}$ before incubation overnight at $37^{\circ} \mathrm{C}$ with 1:200 anti-SCP-3 rabbit antibody (a kind gift from C. Höög, Karolinska Institute, Stockolm). FITC-conjugated antirabbit IgG were used as secondary antibodies.

\section{Identification of apoptotic oocytes by Hoechst staining and TUNEL cytochemistry}

In order to avoid cell loss during the assay, $50 \mu \mathrm{l}$ cell suspension to be analyzed were mixed with an equal volume of $1.5 \%$ low melting point $\left(37^{\circ} \mathrm{C}\right)$ agarose in PBS. Aliquots $(50 \mu \mathrm{l})$ were smeared onto glass slides and allowed to solidify at room temperature. Slides were fixed with $4 \%$ paraformaldehyde for $10 \mathrm{~min}$, washed with PBS, and treated to identify TUNEL-positive cells according to the protocol of the in situ cell death detection kit, Fluorescein (Roche Diagnostics). Finally, cells were stained with Hoechst 33258 nuclear staining $(1 \mu \mathrm{g} / \mathrm{ml}, 5 \mathrm{~min}$, room temperature). TUNEL-positive oocytes were scored in several fields for a total of at least 300 cells under a $40 \times$ objective of a Zeiss Axioplan 2 microscope equipped with a FITC and u.v. fluorescent filter combination.

\section{Transmission electron microscopy (TEM) analysis}

Freshly isolated or cultured ovarian cell population enriched in oocytes by the EDTA-stab method (see above) were centrifuged, washed in PBS, and fixed for $1 \mathrm{~h}$ in $2 \%$ glutaraldehyde in $0.1 \mathrm{M}$ sodium cacodylate buffer ( $\mathrm{pH}$ 7.3). They were then washed thrice with this buffer, post-fixed with $1 \%$ osmium tetroxide aqueous solution, and pre-stained en bloc with a solution of $2 \%$ uranyl acetate in 30\% ethanol. Cells were dehydrated in ethanol, cleared in toluene, and embedded in Epon 812.
Sections were cut at $0.1 \mu \mathrm{m}$ with a LKB Ultrome $V$ and attached onto 150-mesh copper grids pre-treated with Formvar. Grids were stained with lead hydroxide and observed under a $\mathbf{S 7 0 0 0}$ Hitachi transmission electron microscopy.

\section{DNA ladder}

Freshly isolated or cultured ovarian cell population enriched in oocytes were centrifuged, washed in PBS, and lysed overnight at $37^{\circ} \mathrm{C}$ in $1 \mathrm{ml}$ solution containing $200 \mathrm{mM} \mathrm{NaCl}, 20 \mathrm{mM}$ EDTA, $40 \mathrm{mM}$ Tris- $\mathrm{HCl}$ (pH $8.00), 0.9 \%$ SDS, and $400 \mu \mathrm{g} / \mathrm{ml}$ proteinase $\mathrm{K}$. The cell lysates were precipitated with saturated $\mathrm{NaCl}(6 \mathrm{M})$ and $100 \%$ ethanol $(2: 1, \mathrm{v} / \mathrm{v})$ added to supernatants to precipitate DNA. Samples were resuspended in $\mathrm{H}_{2} \mathrm{O}$ and stored at $-20^{\circ} \mathrm{C}$. For autoradiographic analysis, DNA samples $(0.4 \mu \mathrm{g})$ were labeled at the $3^{\prime}$-end with $\left[\alpha^{32} \mathrm{P}\right]$ dideoxy-ATP $(4000 \mathrm{Ci} / \mathrm{mmol})$ by incubation for $60 \mathrm{~min}$ at $37^{\circ} \mathrm{C}$ in the presence of $25 \mathrm{IU}$ terminal transferase (Roche Applied Science). Labeled DNA was electrophoresed ( $0.4 \mu$ g per lane) by a $2 \%$ agarose gel. Gels were dried in a slab-gel dryer for $2 \mathrm{~h}$ and exposed to Kodak X-ray films for $1-3 \mathrm{~h}$ at $-80{ }^{\circ} \mathrm{C}$.

\section{Annexin $V$ binding and evaluation of in situ caspase activity}

Annexin $\mathrm{V}$ binding and caspase activity assay were performed on living morphologically identifiable oocytes using the Annexin V-FITC detection kit (Calbiochem) and the CaspACE FITC-VAD-FMK in situ marker (Promega) respectively, following the protocols of the manufacturers. Annexin V-and caspase-positive oocytes were scored in several fields for a total of at least 300 cells under a $40 \times$ objective of a Zeiss Axioplan 2 microscope equipped with a FITC fluorescent filter combination.

Isolation of apoptotic oocytes was carried out using the Annexin $\mathrm{V}$ Microbead kit from Miltenyi Biotechnology (catalogue no. 130-090-201) as described (Lobascio et al. 2007).

\section{RT-PCR and Western blotting}

Reverse transcriptase PCR (RT-PCR) and immunocytochemistry were used in order to analyze the expression of C-Kit, IGF-I-R, and LIF-R. Briefly, total RNA was extracted from ovarian cell population enriched in oocytes by the RNAzol kit, as described in Pesce et al. (1997). RT-PCR was performed on $1 \mu \mathrm{g}$ aliquot of RNA containing an excess of yeast tRNA as a carrier. RNA was reverse transcribed by Moloney murine leukemia virus reverse transcriptase (Perkin-Elmer, Waltham, MA, USA) using oligo-dT as a primer. Specific cDNAs were then amplified using Ampli-Taq DNA polymerase using 
primers for mouse as follows: c-Kit (385 bp): senseGGCCCAGAGCAAGAGAGGTATCC, antisense-GCCACGATTTCCCTCTCAGC; IGF-IR (395 bp): senseGCAAGTTCTTCGTTTCGTCAT, antisense-CGTAAGGCTGTCTCTCATCAA; LIFR (327 bp): sense-TTCCATTCTTGTCACCCATTA, antisense-ATCTTTACCACTCAGCATTGT; and $\beta$-actin (Nichols et al. 1998) as an internal control.

For Western mini-blotting, cell samples were solubilized in Laemmli buffer and kept frozen at $-80{ }^{\circ} \mathrm{C}$ until use. About $20-30 \mu$ g protein were separated on $10-15 \%$ SDS-PAGE and transferred to nitrocellulose using BioRad transfer apparatus. Blots were blocked for $1 \mathrm{~h}$ at room temperature or overnight at $4{ }^{\circ} \mathrm{C}$ in PBS, $0.1 \%$ Tween 20, and 5-10\% milk. The blocked blots were then probed with the primary antibody (IGF-I receptor (IGF-R) $\alpha$, rabbit-activated caspase- 3 and -6 or mouse caspase- 2 antibodies; Cell Signaling Technology, Danvers, MA, USA or anti-PARP antibody; Santa Cruz, Heidelberg, Germany) diluted 1:1000 in PBS, $0.1 \%$ Tween 20, and $5 \%$ BSA. After washing, the blots were probed for $1 \mathrm{~h}$ with horseradish peroxidase-conjugated secondary antibody (Amersham). After washing, the blots were incubated with ECL chemiluminescent substrate (Amersham) and exposed to film according to manufacturer's instructions.

Densitometric analysis of western bands was performed with ImageQuant software (Molecular Dynamics, Sunnyvale, CA, USA).

\section{Statistical analysis}

Data are expressed as mean \pm s.E.M. of at least three independent experiments with three replicates per experimental group. Comparisons were made by oneway ANOVA, $P$ values $<0.05$ were considered significant.

\section{Results}

In vitro culture of isolated oocytes from 13.5 and $\mathbf{1 5 . 5}$ dpc ovaries: meiotic stage dependence of oocyte death

In a first series of experiments, we analyzed the occurrence of apoptosis in oocytes isolated from fetal ovaries at early $(13.5 \mathrm{dpc})$ and middle $(15.5 \mathrm{dpc}) \mathrm{MPI}$ and cultured for 4 days.

Every day of culture, samples of oocytes identified by morphological features or GCNA-1 staining were analyzed for both meiotic stage and apoptotic features. The results of the Hoechst and TUNEL staining and meiotic spread immunostaining with SCP-3 showed that $13.5 \mathrm{dpc}$ oocytes progressed into meiosis during 4 days of culture with a moderate increase of the percentage of oocytes showing TUNEL staining (Fig. 1A; Fig. 6A, D and $\mathrm{G}, \mathrm{H})$. On the contrary, the meiotic progression of 15.5 dpc oocytes was associated at days 3-4 (T3-T4) of

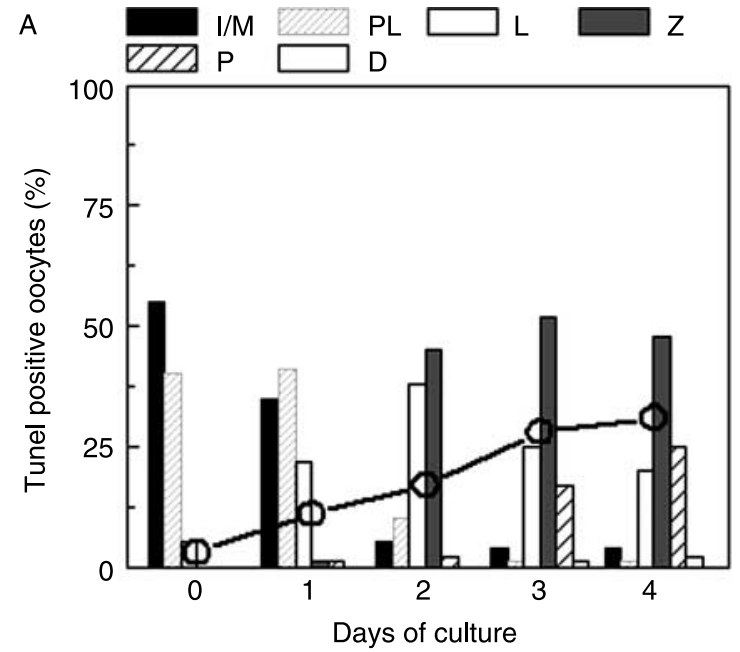

B

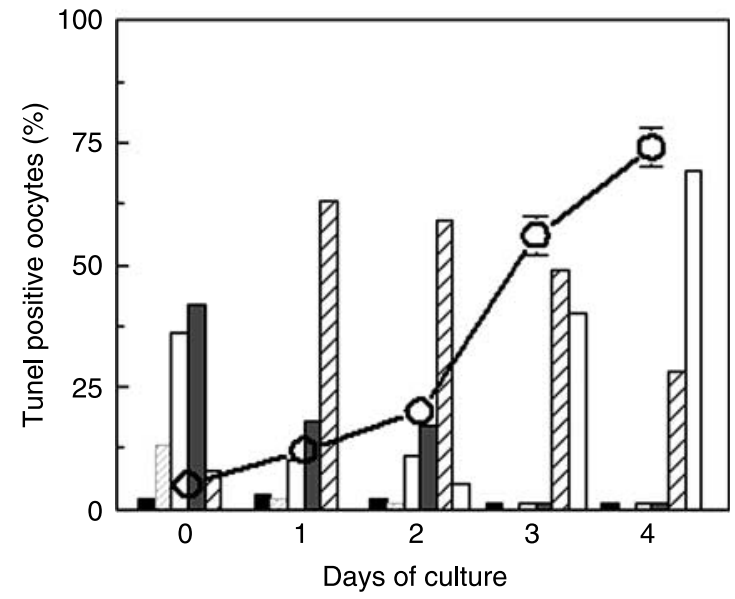

Figure 1 Meiotic stage dependence of oocyte death in culture. Meiotic stages and TUNEL staining evaluated in $13.5 \mathrm{dpc}(\mathrm{A})$ and $15.5 \mathrm{dpc}(\mathrm{B})$ oocytes cultured for 4 days. In 13.5 dpc oocytes, a slight increase in the number of TUNEL-positive oocytes occurred at T3 and T4, whereas in 15.5 dpc oocytes a marked increase takes place at these times. Values are mean \pm S.E.M. of three independent experiments. For meiotic stages, the results of representative experiments are shown. I/M, interphase/mitosis; $\mathrm{PL}$, preleptotene; L, leptotene; $\mathrm{Z}$, zygotene; $\mathrm{P}$, pachytene; $\mathrm{D}$, diplotene.

culture with a marked increase of the number of oocytes positive to the TUNEL staining (Figs 1B, 6A and D). However, as shown in Fig. 1, the percentage of apoptosis estimated at T2 in $13.5 \mathrm{dpc}$ oocytes is constantly higher than that found in oocytes of comparable in vivo age (T0, $15.5 \mathrm{dpc}$ ), indicating that the in vitro condition may exacerbate the apoptotic process.

TUNEL positivity was usually associated with apoptotic morphology (reduced size and condensed chromatin). At all stages analyzed, a fraction of TUNEL-positive oocytes ranging from 10 to $15 \%$ with apparent normal morphology was also observed (data not shown).

In order to verify that the peak of cell death observed at T3-T4 of culture by the TUNEL assay in 15.5 dpc oocytes was due to a time-dependent activation of apoptosis and not to accumulation of degenerated oocytes during the 
culture, apoptotic cells were removed from the medium every day of culture using the Annexin $\mathrm{V}$ binding assay (see below and Materials and Methods). The results confirmed that oocyte apoptosis was low during the first 2 days (around $5-10 \%)$ and underwent a peak (35-50\%) after 3 and 4 days of culture.

\section{TEM morphology of cultured oocytes}

Isolated $15.5 \mathrm{dpc}$ oocytes at the beginning and after 3 days of culture were prepared for morphological TEM observations. Examination at the ultrastructural level of the cultured oocytes allowed us to identify four main types of morphology. Oocytes displaying healthy appearance with no sign of degeneration (Fig. 2A). Oocytes with some characteristics of apoptosis, including chromatin condensation, size shrinkage, and reasonably well-conserved cytoplasmic organelles (Fig. 2B). However, chromatin margination along the nuclear membrane and nucleus fragmentation were never observed. Oocytes showing highly condensed chromatin associated with severely damaged cytoplasmic structures (Fig. 2C). Finally, oocytes exhibiting vacuolated cytoplasm and membrane-bound vacuoles, typical of autophagy, and moderated condensed chromatin (Fig. 2D). Although under TEM observation a precise quantification of these morphologies was not possible, it was apparent that the frequency of the last three types and the presence of hybrid morphology that encompasses all classes described above increased in function of the culture time.

\section{Exposure of phosphotidylserine on the cell membrane of cultured oocytes is associated with TUNEL staining positivity}

In many cell types, exposure of phoshotidylserine (PS) on the outer cell lipid bilayer is a typical early event of apoptosis. Since in fetal oocytes, no information about this apoptotic feature was available, we investigated whether it occurred in cultured oocytes. FITC-Annexin $V$ was used to determine such PS transition. We found that the number of oocytes showing Annexin $\mathrm{V}$ binding in culture parallels that of TUNEL-positive oocytes with a slight more rapid increase during the first 2 days of culture (Figs 3A, 6B and E). Like TUNEL staining, Annexin $\mathrm{V}$ binding was usually associated with morphological characteristics of apoptosis, although some Annexin V-positive oocytes with no apparent morphological features of apoptosis were also observed.

\section{Cultured oocytes undergo oligonucleosomal DNA and PARP-1 cleavage}

Autoradiographic analysis of DNA extracted from 15.5 $\mathrm{dpc}$ ovarian cell populations enriched in oocytes at the
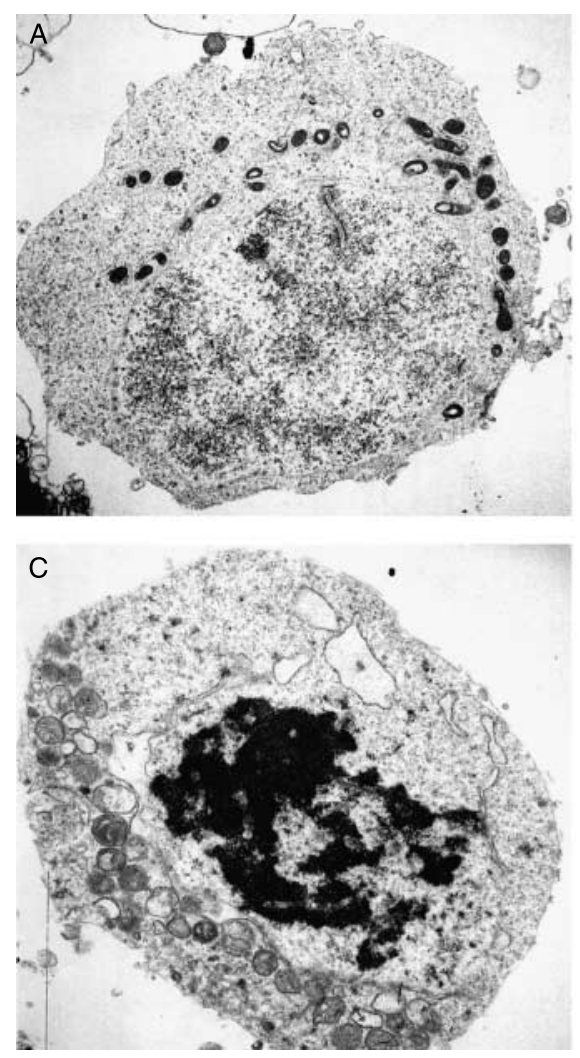
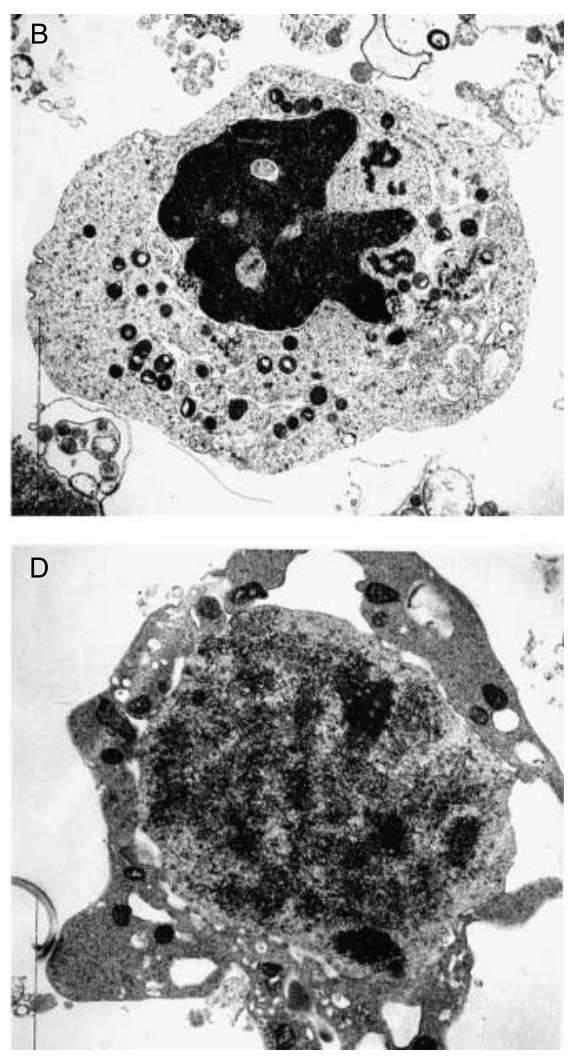

Figure 2 TEM pictures of 15.5 dpc oocytes cultured for 3 days. (A) Oocyte displaying a healthy appearance with no sign of degeneration. (B) Oocyte with apoptosis features including chromatin condensation, size shrinkage, and reasonably well-conserved cytoplasmic organelles. (C) Oocyte showing highly condensed chromatin associated with severely damaged cytoplasmic structures. (D) Oocyte exhibiting vacuolated cytoplasm and membrane-bound vacuoles typical of autophagy and moderated condensed chromatin. A and B 7000, (C) and (D) 9000. 


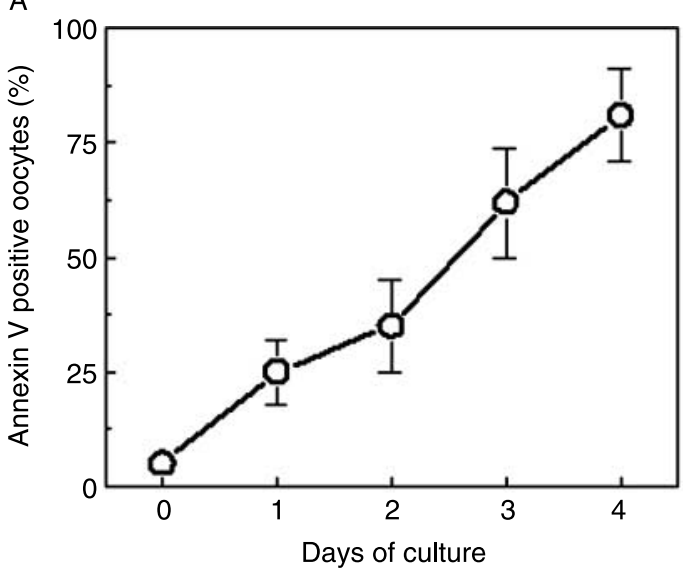

B

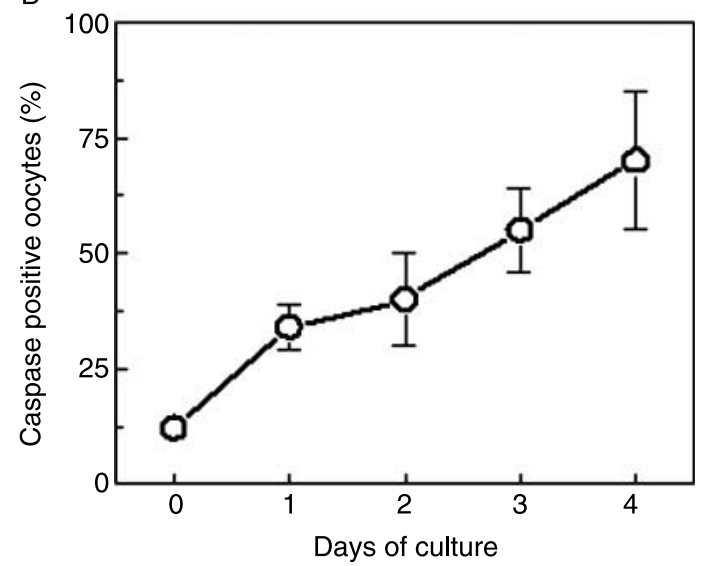

Figure 3 Annexin $\mathrm{V}$ binding (A) and caspase activity (B) in $15.5 \mathrm{dpc}$ oocytes in culture. Values are mean \pm s.E.M. of three independent experiments.

beginning and after 3 days of culture showed in the latter the presence of oligonucleosomal DNA fragments typical of apoptosis (Fig. 4A). Similarly, up to two- to threefold increase of the cleaved forms of the nuclear protein poly(ADP-ribose) polymerase (PARP)-1 into $89 \mathrm{kDa}$ and $24 \mathrm{kDa}$ fragments, generally associated with the activation of caspase- 3 and -7 during apoptosis (Ruscetti et al. 1998, Los et al. 2002), was evident in protein extract from the 3-day cultured cells (Fig. 4B).

\section{Caspase activity in cultured oocytes}

Caspase activity was detected in single cells using a cellpermeable fluorogenic caspase-specific peptide substrate (CaspACE FITC-VAD-FMK) that fluoresces once cleaved (see Materials and Methods). The pattern of caspase activation in cultured oocytes was substantially similar to that of Annexin $\mathrm{V}$ binding and roughly parallels that of TUNEL staining (Figs 3B, 7C and F). However, at T3 and T4 a subpopulation of TUNELpositive oocytes $(\sim 10-20 \%)$ resulted caspase negative.

Western blotting analyses in fetal oocytes of the expression of three effector caspases such as caspase-3,
A

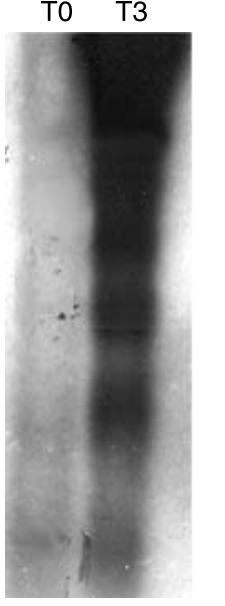

B
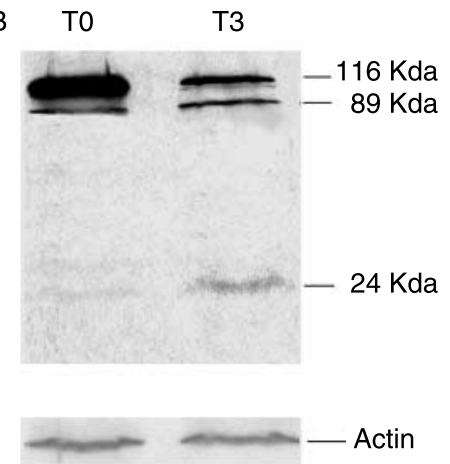

Figure 4 Detection of molecular markers of apoptosis in $15.5 \mathrm{dpc}$ oocytes in culture. (A) Gel electrophoresis of DNA cleavage in oligonucleosomal fragments (DNA ladder) in oocytes at the beginning (T0) and after 3 days (T3) of culture. (B) Western blotting of PARP-1 cleavage during oocyte culture showing a decrease of $116 \mathrm{kDa}$ band intensity and appearance of $24 \mathrm{kDa}$ band were at T3. These experiments were repeated at least twice.

-6 , and -2 revealed that only the latter was present in these cells (Fig. 5, data not shown) and resulted upregulated and cleavage activated in oocytes undergoing apoptosis in culture (Fig. 5).

\section{Oocyte apoptosis vitro is not influenced by serum but can be partly prevented by caspase inhibitors, $K L$ and IGF-I growth factor and $\mathrm{N}$-acetyl-L-cysteine (NAC)}

The continuous presence in the culture medium of the broad-spectrum caspase inhibitor Z-VAD-FMK at a concentration which in preliminary experiments (data not shown) we had found not toxic for the oocytes $(10-50 \mu \mathrm{M})$ resulted in the reduction of the number of oocytes showing caspase activity (mean \pm S.E.M., T3: control $60 \%+3.2$ versus Z-VAD-FMK $45 \%+4.6$ ) associated with a significant attenuation of oocyte showing TUNEL staining (T3: control $=62 \% \pm 5.5$ versus Z-VAD-FMK $=35 \% \pm 1.5$; T4: control $=76 \% \pm 9.5$ versus $Z-V A D-F M K=65 \pm 3.5$ ). At T4, however, such decrease was not statistically significant. Replenishing the medium every day with half the concentration of inhibitor did not significantly change the results (data not shown). Similar results were obtained using the caspase2-specific inhibitor Z-VDVAD (data not shown), confirming the main role of such caspase in this process.

It has been previously shown that KL and other growth factors (see above) are able to partly prevent apoptosis of primordial germ cells and of isolated and/or ovaryenclosed fetal oocytes as detected by cell morphology and histochemical markers (Pesce et al. 1993, Pesce \& De Felici 1994, De Felici et al. 1999, Morita et al. 1999, 2001). Moreover, Morita et al. (1999) reported that the rate of oocyte degeneration in cultured embryonic 


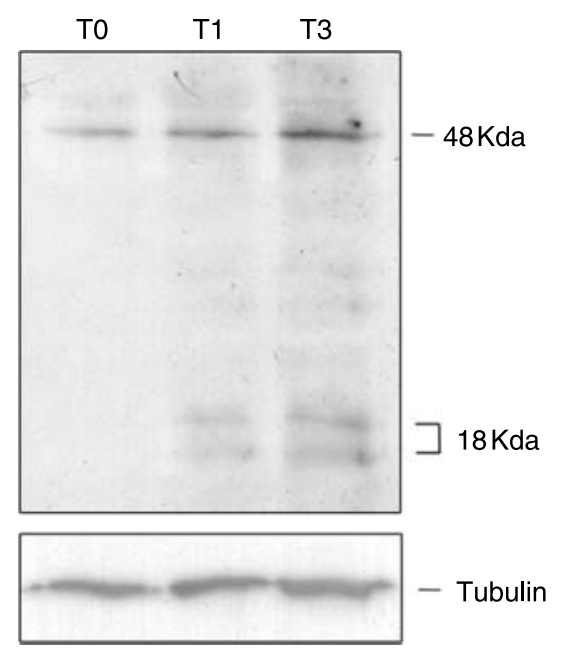

Figure 5 Western blotting analysis of caspase-2 expression in $15.5 \mathrm{dpc}$ oocytes. While in oocytes at T0 only procaspase-2 was detectable, cleaved caspase-2 forms indicative of enzyme activation appeared at $\mathrm{T} 1$ and T3. Moreover, densitometric analysis showed that at these culture times, the expression of caspase- 2 was increased. These experiments were repeated twice.

ovaries was not affected by the presence of serum. In order to further characterize oocyte death in our culture system and to verify whether the system is amenable to be manipulated to achieve information on the mechanisms of this process, we tested the effect of serum of some of the above-mentioned growth factors, such as KL, IGF-I and LIF, and of the antioxidant NAC on oocyte death evaluated by the TUNEL assay. The results showed that oocyte apoptosis in culture was not significantly influenced by the presence of serum (5\% HS plus $2.5 \%$ FCS; TUNEL-positive oocytes at T3: + serum $=71 \% \pm 7.5$ versus - serum $=60 \pm 4.5$; at $\mathrm{T} 4:+$ serum $=82 \% \pm 7.5$ versus - serum $=78 \% \pm 4.5$; $P<0.05)$, and that with or without serum the presence of $100 \mathrm{ng} / \mathrm{ml} \mathrm{KL}$ reduced significantly the number of TUNEL-positive oocytes at T3 and T4 (Fig. 8A). The same analysis of cultured oocytes showed that $100 \mathrm{ng} / \mathrm{ml}$ IGF-I was able to reduce oocyte apoptosis to a similar extent (Fig. 8A), while the addition to the culture medium of $1000 \mathrm{IU} / \mathrm{ml}$ LIF did not affect the number of TUNEL-positive oocytes (data not shown). The effect of $\mathrm{KL}$ and IGF-I was not additive (data not shown).

Furthermore, we found that mRNA for IGF-R was expressed at similar levels in ovarian cell population enriched in oocytes at all stages examined, whereas transcripts for the low affinity LIF receptor (LIF-R) were not detectable (Fig. 7B). The presence of IGF-R also at protein level was confirmed by Western blotting (data not shown). As expected (Manova \& Bachvarova 1991, De Felici et al. 1996), ovarian cell population enriched in oocytes obtained from 16.5 to $18.5 \mathrm{dpc}$ embryos expressed increasing levels of transcripts for the $\mathrm{KL}$ receptor C-Kit (Fig. 7B).
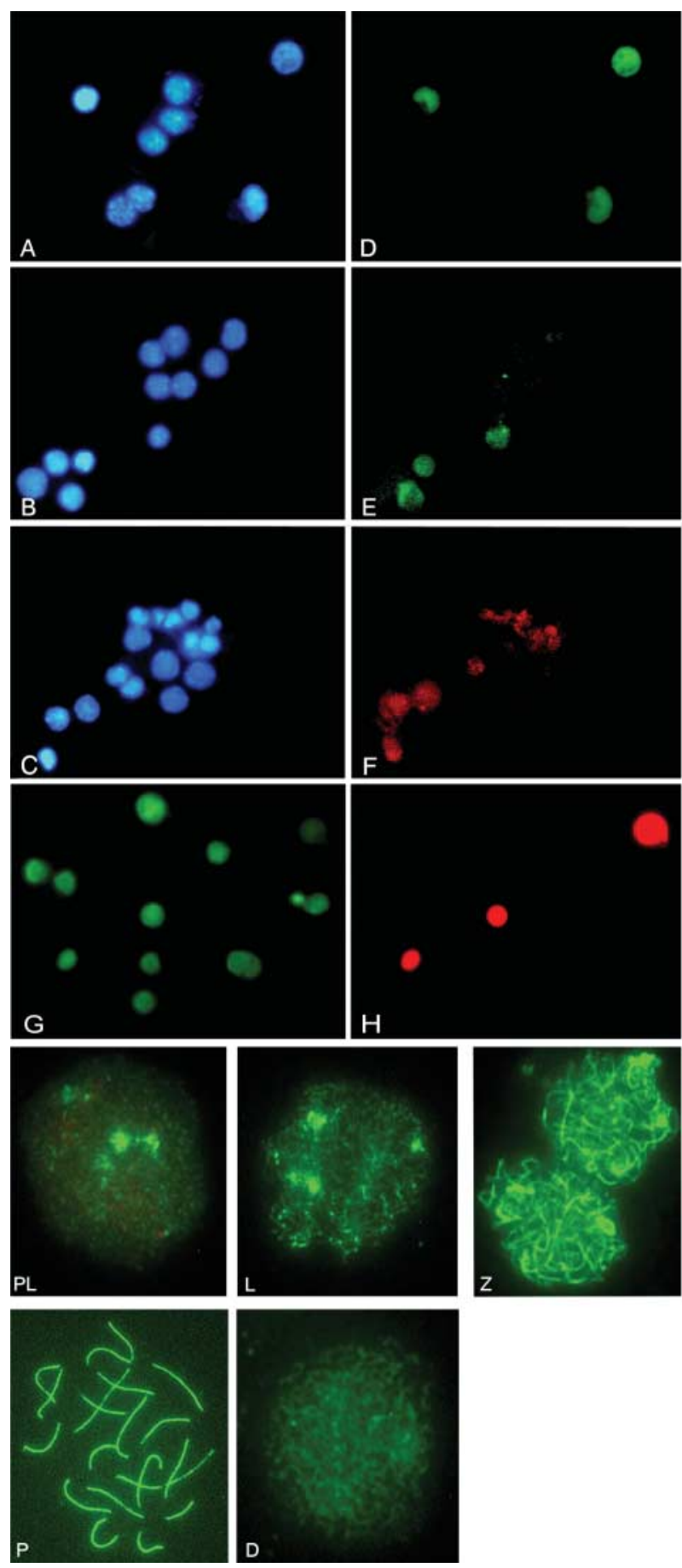

Figure 6 Identification of oocyte apoptosis and meiotic stages by various markers. (A-C) Hoechst 33258-labeled 15.5 dpc oocytes in culture and (D-F) the same oocytes after TUNEL, Annexin-V and CaspACE FITC-VAD-FMK staining. (G) GCNA1-labeled oocytes, (H), the same oocytes after TUNEL staining. The lower pictures show stages of meiotic prophase I as identified by SCP-3 labeling. PL, preleptotene; L leptotene; Z, zygotene; P, pachytene; D, diplotene. 
A

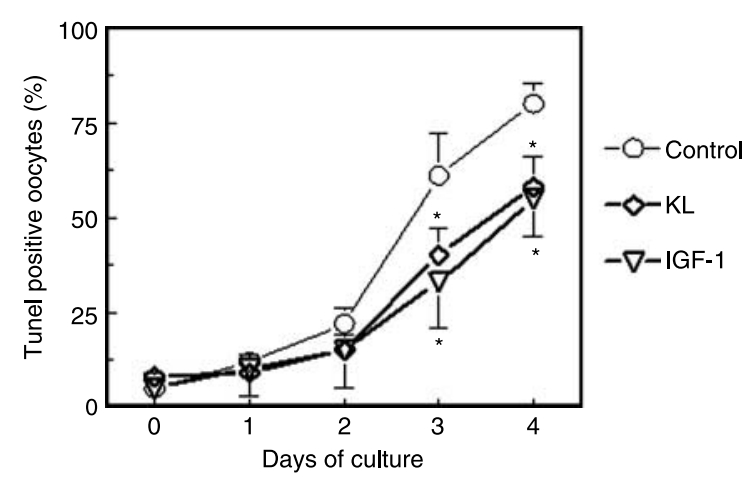

B

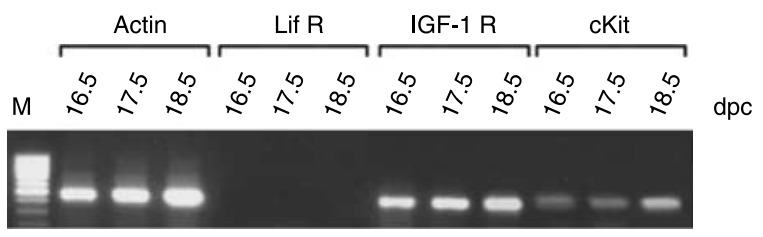

Figure $7 \mathrm{KL}$ and IGF-I partly prevent oocyte apoptosis in culture. (A) Effect of $100 \mathrm{ng} / \mathrm{ml} \mathrm{KL}$ or $100 \mathrm{ng} / \mathrm{ml} \mathrm{IGF-I} \mathrm{on} \mathrm{the} \mathrm{number} \mathrm{of} \mathrm{TUNEL-}$ positive oocytes cultured in the medium supplemented with serum. Similar results were obtained in serum-free medium (data not shown). Values are mean \pm S.E.M. of three independent experiments. Asterisks indicate statistically significant difference in comparison with control. (B) RT-PCR analysis of the expression of mRNA for c-Kit, IGF-R, and LIF$\mathrm{R}$ in oocytes obtained from 16.5 to $18.5 \mathrm{dpc}$ ovaries.

Antioxidant defenses play a critical role in the regulation of $P C D$, even when death is induced by nonoxidative stimuli. In this regard, we found that the addition to the culture medium of increasing concentration of NAC, a well-established thiol antioxidant, resulted in significant reduction of the number of TUNEL-positive oocytes at T3. As for caspase inhibitors, however, at T4 this effect was transient and not statistically significant (Fig. 8).

\section{Oocyte apoptosis is reduced by calpain inhibitor I and increased by rapamycin after prolonged culture}

Since the results reported above suggested that oocyte degeneration may occur by alternative types of PCD, we tested whether calcium-activated neutral proteases (calpains) and mammalian target of rapamycin (mTOR), typically involved in various types of cell death (for reviews, see Castedo et al. 2002, Bröker et al. 2005), contribute to such process. We found that the addition of $5 \mu \mathrm{M}$ calpain inhibitor I ALLN to the culture medium significantly reduced the level of oocyte apoptosis only at T4 (Fig. 9), whereas $50 \mathrm{nM}$ rapamycin increased apoptosis both at T3 and T4 (Fig. 10). In addition, when rapamycin was used together with $\mathrm{KL}$ the anti-apoptotic effect of this growth factor was not affected, while the increased apoptosis induced by rapamycin was abolished (Fig. 10).

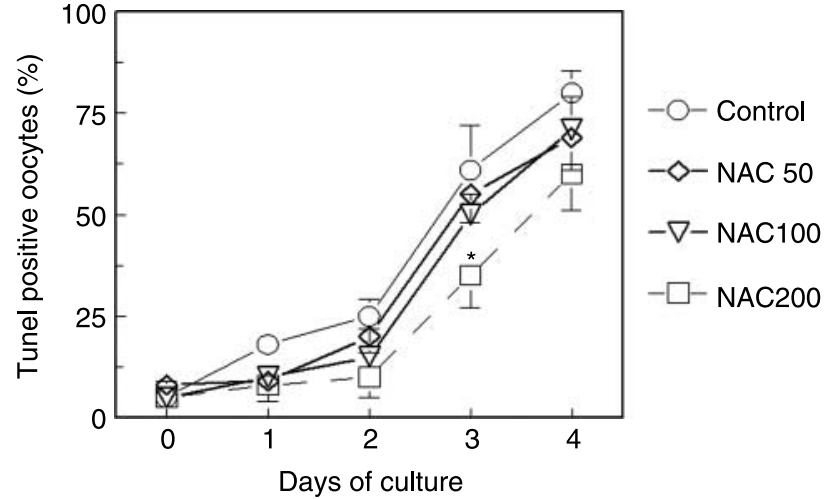

Figure 8 Effect of $\mathrm{N}$-acetyl-L-cysteine (NAC) on oocyte apoptosis. Reduction of the number of TUNEL-positive 15.5 dpc oocytes in culture by increasing concentrations of NAC. Values are mean \pm S.E.M. of three independent experiments. Asterisks indicate statistically significant difference in comparison with control.

\section{Discussion}

Previous studies suggested that in mammals the major reduction of oocyte numbers occurring in the fetal ovary during MPI is caused by PCD processes. In the present paper, in order to determine the characteristics and mechanisms of these processes, we used a culture system that allows isolated mouse oocytes to progress in vitro throughout $\mathrm{MPI}$.

In a first series of experiments, we found that the TUNEL assay was able to identify most of the oocytes undergoing degeneration in culture. We found that the number of TUNEL-positive oocytes increased in culture as a function of the meiosis progression; the frequency of TUNEL-positive oocytes is low at early stages of MPI and peaks when they reach the pachytene/diplotene stage.

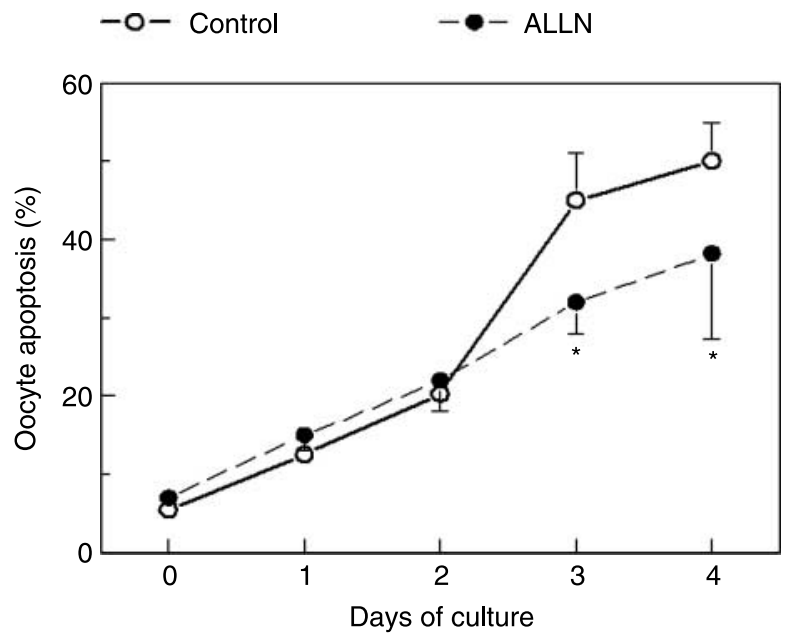

Figure 9 Effect of $5 \mu \mathrm{M}$ calpain inhibitor I ALLN on oocyte apoptosis. Reduction of the number of TUNEL-positive 15.5 dpc oocytes in culture at T4. Values are mean \pm S.E.M. of three independent experiments. Asterisks indicate statistically significant difference in comparison with control. 


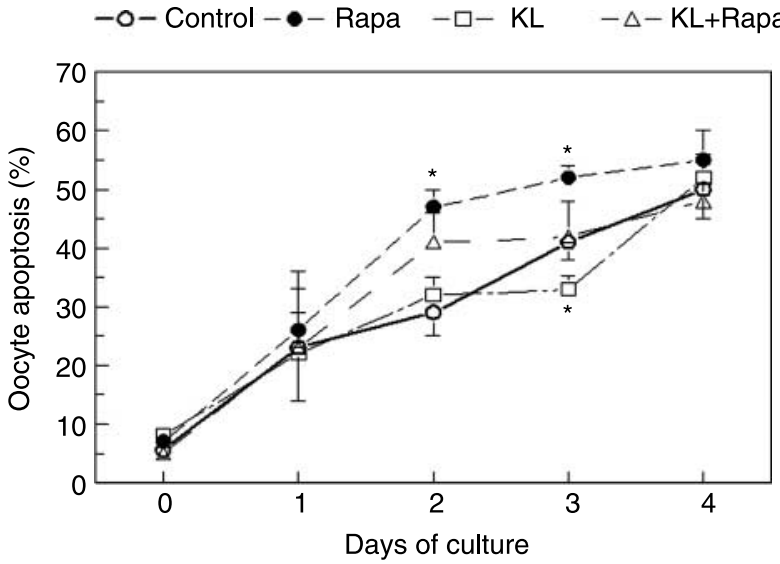

Figure 10 Effect of $50 \mathrm{nM}$ rapamycin (Rapa) on oocyte apoptosis. Increase of the number of TUNEL-positive $15.5 \mathrm{dpc}$ oocytes in culture at T3 and T4. Note that Rapa did not affect the anti-apoptotic effect of $100 \mathrm{ng} / \mathrm{ml} \mathrm{KL}$, while this latter abolished the anti-apoptotic effect of Rapa. Values are mean \pm s.E.M. of three independent experiments. Asterisks indicate statistically significant difference in comparison with control.

Previous studies performed in vivo by us (Pesce et al. 1997) and others (Pepling \& Spradling 2001) have shown that TUNEL-positive oocytes ranging from 0.5 to $5 \%$ of the total germ cell population are present in the mouse fetal ovaries from $13.5 \mathrm{dpc}$ to early post natal ages. Considering that apoptosis is a relatively rapid process characterized by rapid removal of dead cells (from $1 \mathrm{~h}$ to 5-6 h), these percentages are consistent with $60-70 \%$ oocyte depletion estimated in several studies (Borum 1961, Bakken \& McClanahan 1978, McClellan et al. 2003) and near to those found by us at T3/T4 of culture of $15.5 \mathrm{dpc}$ oocytes in a condition in which apoptotic oocytes are not readily removed.

While in vivo observations about the stage dependence of the PCD process are contradictory (see Introduction), our in vitro observations support the notion that oocyte PCD depends at least, in part, on the oocyte-autonomous activation of meiotic checkpoints occurring preferentially at certain stages of MPI. Meiotic checkpoints operating at the pachytene stage that prevent meiotic progression when defects in chromosome recombination and/or synapsis occur have been described in several species including mammals (for a review, see Roeder \& Bailis 2000). As reported in the Introduction, knockout mice have been generated in which spermatocytes and oocytes arrest at mid-meiotic prophase for defects in the chromosome crossover. In such mutant mice, germ cells arrest in meiotic prophase and are thought to undergo apoptosis following meiotic checkpoint activation (Pittman et al. 1998, Yoshida et al. 1998, Edelmann et al. 1999, de Vries et al. 1999, Kneitz et al. 2000). A large number of proteins are likely involved in the meiotic checkpoint including ATM, ATR, BRCA1, $\mathrm{H}_{2} \mathrm{AX}$, and MDC1 (Keegan et al. 1996, Barlow et al. 1998, Turner et al. 2004, Lee et al. 2005), but in mammal fetal oocytes their precise role(s) in this process and whether and how they operate in triggering apoptosis during MPI remain to be determined.

The culture of oocytes described here allowed also to establish for the first time that PS exposure on the outside membrane (detected by Annexin $\mathrm{V}$ binding), oligonucleosome DNA (DNA laddering), and PARP-1 cleavage are events associated with fetal oocyte death. All these processes are classical biochemical markers of apoptosis. It is worthy to mention that the capability of apoptotic oocytes to bind Annexin $\mathrm{V}$ allowed us also to devise a simple and rapid method to isolate apoptotic from nonapoptotic fetal oocytes (Lobascio et al. 2007). A major problem in studying the mechanisms underlying cell apoptosis is to obtain sufficient quantities of homogenous experimental material for biochemical analyses. If major events of oocyte apoptosis can be recapitulated in vitro, as suggested by the present results, the possibility to isolate apoptotic and non-apoptotic oocytes in culture should make it easier to overcome this difficulty.

Another common event in classical apoptosis is the activation of a family of cysteine proteinases termed caspases. The involvement of caspase- 2 in the execution of oocyte apoptosis has been demonstrated by Bergeron et al. (1998). These authors showed that the ovaries of 3- to 4-day-old female caspase-2-deficient mice possess a surplus of primordial follicles. Our results confirm and expand these early results showing that also oocyte death in culture is accompanied by caspase activation, namely caspase-2, and that a broad-spectrum caspase inhibitor (Z-VAD-FMK) and the caspase-2-specific inhibitor Z-VDVA-FMK as well may exert a significant albeit transitory protective effect on this process.

Although our results indicate that most of the oocytes in culture undergo cell death with hallmarks of apoptosis, our new observations indicate that this process appears in some instance morphologically atypical and may be distinctly different from classical apoptosis. In fact, TEM observations showed a variety of oocyte death morphologies including oocytes with no evidence of chromatin margination and nucleus fragmentation and oocytes with autophagic and necrotic features. While the necrotic morphologies are almost certainly due to secondary necrosis occurring in dying oocytes not removed from the culture, the lack of some features of the classically described apoptosis might be due to a unique structure of the germ cell chromatin and specifically of meiotic germ cells. Lack of typical apoptosis morphology has been reported in degenerating mouse spermatogonia around birth and in rat postnatal follicle-enclosed oocytes (Wang et al. 1998, Devine et al. 2000). While it is possible that some forms of oocyte death observed by us are a consequence of the culture condition, we favor the hypothesis that alternatively or in addition to apoptotic degeneration fetal 
oocytes might follow distinct forms of PCD including autophagic and caspase-independent processes depending on the apoptotic stimulus and the cellular context. In this regard, it is worthy to be pointed out that at T3 and T4 we observed a significant number of TUNEL-positive oocytes (ranging from 10 to 20\%) lacking significant caspase activity.

Other evidence coming from our data support previous results showing that death of fetal oocytes partly depends on limited availability of growth factors. In accord with Morita et al. (1999), who used cultures of fetal ovaries, we found that the presence of serum in the culture medium did not significantly affect oocyte death, while the addition of KL or IGF-I significantly decreased the number of TUNEL-positive oocytes in culture. Unlike Morita et al. (1999), however, we did not find a significant anti-apoptotic effect of LIF on isolated oocytes. In line with these observations, we found that oocytes express transcripts for c-Kit (the KL receptor) and IGF-IR while they do not express mRNA for the low affinity LIF-R. Since a low amount of LIF-R mRNA was detectable in the whole fetal ovary (data not shown), it is possible that LIF may exert an indirect anti-apoptotic effect on the fetal oocyte through somatic cells.

The intracellular signaling activated by c-Kit and IGF-I in fetal oocytes remains to be investigated. In addition, an intriguing possibility to address in future studies is whether growth factors can influence some events of crossover in fetal oocytes by acting on the expression and/or the activity of the involved proteins. In this regard, two recent papers showed that cytokines are able to induce the expression of the nucleotide excision repair genes in human keratinocytes (Schwarz et al. 2001) and that insulin can activate ATM (Yang \& Kastan 2001), a key enzyme in DNA damage repair, in mouse fibroblasts.

In a further series of experiments, we chose NAC as a potential inhibitor of fetal oocyte apoptosis in culture because it has been shown to suppress apoptosis in cultured rat and human ovarian follicles (Tilly \& Tilly 1995, Otala et al. 2002) as well as in other gonadal cell types (Erkkila et al. 1998, Lee et al. 2000, Farini et al. 2003). NAC is a well-established thiol antioxidant that, after uptake, deacetylation, and conversion to glutathione, functions as both a redox buffer and a reactive oxygen intermediate scavenger. We observed up to $30 \%$ inhibition of oocyte apoptosis in culture at a concentration of $200 \mu \mathrm{g} / \mathrm{ml}$ NAC. Thus, oxidative stress may play a role at least in vitro in fetal oocyte apoptosis. However, the exact mechanism underlying such NAC action remains to be elucidated since NAC encompasses an impressive array of potential targets of action other than those related to its antioxidative function.

Eventually, since the results discussed above suggested that oocyte degeneration may occur by alternative types of PCD, we tested whether calpains and mTOR were involved in such other pathways. Calpains have been shown to participate in various types of cell death, including both caspase-dependent and caspase-independent pathways (for reviews, see Bröker et al. 2005), while mTOR acts as pleiotropic cell death regulator mainly involved in the negative control of autophagy (for a review, see Castedo et al. 2002). The anti-apoptotic effect by the calpain inhibitor I, which we evidenced only after 4 days of culture, indicates that calpains play a role in oocyte apoptosis in culture, likely in cell death pathways activated after prolonged culture, when we observed increased number of atypical apoptotic morphologies and TUNEL-positive and caspase-negative oocytes. Indeed, it has been shown that both caspases and calpains induce release of lysosomal cathepsin and subsequent autophagia (Sanvincens et al. 2004). Moreover, calpains play a key role in other types of caspaseindependent cell death in various cell types (for a review, see Bröker et al. 2005). mTOR is a well-known downstream effector of the phosphotidylinositol/protein kinase B (Akt) signaling pathways and a central modulator of cell proliferation, growth, and differentiation. In addition, mTOR can control whether a cell undergoes apoptosis or autophagy (Castedo et al. 2002). Rapamycin specifically inhibits the function of mTOR and induces cell death in a cell-type-specific fashion (for references, see Takeuchi et al. 2005). It is still unclear whether cross-talk occurs between these two PCD pathways and what determines why cells die from one type or the other. In our culture system, rapamycin was able to increase oocyte apoptosis evaluated by the TUNEL staining at T3 and T4 indicating that a basal level of active mTOR is needed to maintain prolonged oocyte survival. Since it is generally accepted that mTOR inhibits autophagy, it is likely that activation of this process is responsible of the increased level of cell death in cultured oocytes caused by rapamycin. Interestingly, the $\mathrm{KL}$ anti-apoptotic action on oocytes does not appear dependent on the downstream activation of mTOR since it is not affected by the presence of rapamycin in the culture medium. On the other hand, KL action appears to abolish the pro-apoptotic effect of rapamycin suggesting cross-talk between these pathways. In their complex, these last results support the notion that overlapping and shared molecular pathways among different death programs can be activated in fetal oocytes depending on the cell contest.

A final speculation rising from our results is about the relative inefficiency of compounds and conditions used in the present paper in preventing oocyte death. Beyond the limits of the culture conditions, this might be consistent with the fact that main role of fetal oocyte PCD is to eliminate poor quality oocytes and, for this reason, must be little amenable to inhibition. In this context, a variety of alternative death ways may assure that when a pathway cannot be properly followed, activation of another leads defective oocytes to degeneration. 


\section{Acknowledgements}

We thank Mr Antonio Volpe for expert technical assistance in the preparation of TEM samples and Dr Ilana Moscatelli for critical reading of the paper. This work was supported by EU QLRT-2001-02403, ASI grant, and MIUR National Project Cofin. The authors declare that there is no conflict of interest that would prejudice the impartiality of this scientific work.

\section{References}

Bakken AH \& McClanahan M 1978 Patterns of RNA synthesis in early meiotic prophase oocytes from fetal mouse ovaries. Chromosoma 67 21-40.

Barlow C, Liyanage M, Moens PB, Tarsounas M, Nagashima K, Brown K, Rottinghaus S, Jackson S, Tagle D, Ried T et al. 1998 Atm deficiency results in severe meiotic disruption as early as leptotene of prophase I. Development 125 4007-4017.

Bergeron L, Perez GI, Macdonald G, Shi L, Sun Y, Jurisicova A, Varmuza S, Latham KE, Flaws JA, Salter JCM et al. 1998 Defects in regulation of apoptosis in caspase-2-deficient mice. Genes and Development 12 1304-1314.

Borum K 1961 Oogenesis in the mouse. A study of the meiotic prophase. Experimental Cell Research 24 495-507.

Bröker LE, Kruyt FAE \& Giaccone G 2005 Cell death independent of caspases; a review. Clinical Cancer Research 11 3155-3162.

Burgoyne PS \& Baker TG 1981 Oocyte depletion in XO mice and their sibs from 12 to 200 days post partum. Journal of Reproduction and Fertility 61 207-212.

Burgoyne PS \& Baker TG 1985 Perinatal oocyte loss in XO mice and its implications for the aetiology of gonadal dysgenesis in $X O$ woman. Journal of Reproduction and Fertility 75 633-645.

Castedo M, Ferri KF \& Kroemer G 2002 Mammalian target of rapamycin (mTOR): pro-and anti-apoptotic. Cell Death and Differentiation 9 99-100.

Devine PJ, Payne CM, McCuskey MK \& Hoyer PB 2000 Ultrastructural evaluation of oocytes during atresia in rat ovarian follicles. Biology of Reproduction 63 1245-1252.

Edelmann W, Cohen PE, Kneitz B, Winand N, Lia M, Heyer J, Kolodner R, Pollard JW \& Kucherlapati R 1999 Mammalian MutS homologue 5 is required for chromosome pairing in meiosis. Nature Genetics 21 123-127.

Enders GC \& May JJ 1994 Developmentally regulated expression of a mouse germ cell nuclear antigen examined from embryonic day 11 to adult in male and female mice. Developmental Biology 163 331-340.

Erkkila K, Hirvonen V, Wuokko E, Parvinen M \& Dunkel L 1998 $\mathrm{N}$-acetyl-L-cysteine inhibits apoptosis in human male germ cells in vitro. Journal of Clinical Endocrinology and Metabolism 83 2523-2531.

Farini D, Puglianiello A, Mammi C, Siracusa G \& Moretti C 2003 Dual effect of pituitary adenylate cyclase activating polypeptide on prostate tumor LNCaP cells: short- and long-term exposure affect proliferation and neuroendocrine differentiation. Endocrinology 144 1631-1643.

De Felici M 2000 Regulation of primordial germ cell development in the mouse. International Journal of Developmental Biology $4 \mathbf{4}$ 575-580.

De Felici M \& McLaren A 1982 Isolation of mouse primordial germ cells. Experimental Cell Research 142 282-476.

De Felici M, Di Carlo A, Pesce M, Iona S, Farrace MG \& Piacentini M $1999 \mathrm{BCl}-2$ and Bax regulation of apoptosis in germ cells during prenatal oogenesis in the mouse embryo. Cell Death and Differentiation 6 908-915.
De Felici M, Klinger FG, Farini D, Scaldaferri ML, lona S \& Lobascio M 2005 Establishment of oocyte population in the fetal ovary: primordial germ cell proliferation and oocyte programmed cell death. Reproductive Biomedicine Online 10 182-191.

Keegan KS, Holtman DA, Plug AW, Christenson ER, Brainerd EE, Flaggs G, Bentley JN, Taylor MK, Meyn MS, Moss SB et al. 1996 The Atr and Atm protein kinases associate with different sites along meiotically pairing chromosomes. Genes and Development $\mathbf{1 0}$ 2423-2437.

Kneitz B, Cohen PE, Avdievich E, Zhu L, Kane MF, Hou H, Kolodner RD, Kucherlapati R, Pollard JW \& Edelmann W 2000 MutS homolog 4 localization to meiotic chromosomes is required for chromosome pairing during meiosis in male and female mice. Genes and Development 14 1085-1097.

Lee AC, Fernadez-Capetillo O, Pisupati V, Jackson SP \& Nussenzeweig A 2005 Specific association of mouse MDC1/NFBD1 with NBS1 at sites of DNA damage. Cell Cycle 4 177-182.

Lobascio AM, Klinger FG \& De Felici M 2007 Isolation of apoptotic mouse fetal oocytes by AnnexinV assay. International Journal of Developmental Biology 51 157-160.

Los M, Mozoluk M, Ferrari D, Stepczynska A, Stroh C, Renz A, Herceg Z, Wang ZQ \& Schulze-Osthoff K 2002 Activation and caspase-mediated inhibition of PARP: a molecular switch between fibroblast necrosis and apoptosis in death receptor signalling. Molecular Biology of the Cell 13 978-988.

Mahadevaiah SK, Turner JMA, Baudat F, Rogakou EP, de Boer P, Blanco-Rodriguez J, Jasin M, Keeny S, Bonner WM \& Burgoyne PS 2001 Recombinational DNA double-strand breaks in mice precede synapsys. Nature Genetics 27 271-276.

Manova K \& Bachvarova R 1991 Expression of c-kit encoded at the W locus of mice in developing embryonic germ cells and presumptive melanoblasts. Developmental Biology 146 312-324.

Matikainen T 2001 Aromatic hydrocarbon receptor-driven Bax gene expression is required for premature ovarian failure caused by biohazardous environmental chemicals. Nature Genetics $\mathbf{2 8}$ 355-360.

McClellan KA, Gosden R \& Taketo T 2003 Continuous loss of oocytes throughout meiotic prophase in the normal mouse ovary. Developmental Biology 258 334-348.

Modi DN, Sane S \& Bhartiya D 2003 Accelerated germ cell apoptosis in sex chromosome aneuploid fetal human gonads. Molecular Human Reproduction 9 219-225.

Morita Y \& Tilly JL 1999 Oocytes apoptosis: like sand through an hourglass. Developmental Biology 213 1-17.

Morita Y, Manganaro TF, Tao XJ, Martinbeau S, Donahoe PK \& Tilly JL 1999 Requirement for phosphatidylinositol-3'-kinase in cytokinemediated germ cell survival during fetal oogenesis in the mouse. Endocrinology 140 941-949.

Morita Y, Maravei DV, Bergeron L, Wang S, Perez GI, Tsutsumi O, Taketani Y, Asano M, Horai R, Korsmeyer SJ et al. 2001 Caspase-2 deficiency prevents programmed germ cell death resulting from cytokine insufficiency but not meiotic defects caused by loss of ataxia telangiectasia-mutated (Atm) gene function. Cell Death and Differentiation 8 614-620.

Nichols J, Zevnik B, Anastassiadis K, Niwa H, Klewe-Nebenius D, Chambers I, Schöler H \& Smith A 1998 Formation of pluripotent stem cells in the mammalian embryo depends on the POU transcription factor Oct4. Cell 95 379-391.

Otala M, Erkkila K, Tuuri T, Sjoberg J, Suomalainen L, Suikkari AM, Pentikainen V \& Dunkel L 2002 Cell death and its suppression in human ovarian tissue culture. Molecular Human Reproduction 8 228-236.

Pepling ME \& Spradling AC 2001 Mouse ovarian germ cell cysts undergo programmed breakdown to form primordial follicles. Developmental Biology 234 339-351.

Perez GI, Robles R, Knudson CM, Flaws JA, Korsmeyer SJ \& Tilly JL 1999 Prolongation of ovarian lifespan into advanced chronological age by Bax-deficiency. Nature Genetics 21 200-203. 
Pesce M \& De Felici M 1994 Apoptosis in mouse primordial germ cells: a study by transmission and scanning electron microscope. Anatomy and Embryology 189 435-440.

Pesce M, Farrace MG, Dolci S \& De Felici M 1993 Stem cell factor and leukemia inhibitory factor promote primordial germ cell survival by suppressing programmed cell death (apoptosis). Development 118 1089-1094.

Pesce M, Farrace MG, Amendola A, Piacentini M \& De Felici M 1997 Stem cell Factor Regulation of apoptosis in mouse primordial germ cells. In Cell Death in Reproductive Physiology, pp 19-31. Eds JL Tilly \& JF Strauss. New York: Verlag.

Pittman DL, Cobb J, Schimenti KJ, Wils LA, Cooper DM, Brignull E, Handel MA \& Schimenti JC 1998 Meiotic prophase arrest with failure of chromosome synapsis in deficient for Dmc1, a germlinespecific RecA homolog. Molecular Cell 1 697-705.

Ratts VS, Flaws JA, Kolp K, Sorenson CM \& Tilly JL 1995 Ablation of $b c l-2$ gene expression decreases the numbers and oocytes and primordial follicles established in the post-natal female mouse gonad. Endocrinology 136 3665-3668.

Roeder GS \& Bailis JM 2000 The pachytene checkpoint. Trends in Genetics 16 395-403.

Rucker EB III, Dierisse P, Wagner KU, Garret L, Wynshaw-Boris A, Flaws JA \& Hennighausen L $2000 \mathrm{BCl}-x$ and Bax regulate mouse primordial germ cell survival and apoptosis during embryogenesis. Molecular Endocrinology 14 1038-1052.

Ruscetti T, Lehnert BE, Halbrook J, Le Trong H, Hoekstra MF, Chen DJ \& Peterson SR 1998 Stimulation of the DNA-dependent protein kinase by poly(ADP-ribose) polymerase. Journal of Biological Chemistry 273 14461-14467.

Sanvincens N, Gomez-Vicente V, Masip I, Messeguer A \& Cotter TG 2004 Oxidative stress-induced apoptosis in retinal photoreceptor cells is mediated by calpains and caspases and blocked by the oxygen radical scavenger CR-6. Journal of Biological Chemistry 279 39268-39278.

Schwarz A, Stander S, Berneburg M, Bohm M, Kulms D, Steeg HV, Grosse-Heitmeyer K, Krutmann J \& Schwarz T 2001 Interleukin-12 suppresses ultraviolet radiation-induced apoptosis by inducing DNA repair. Nature Cell Biology 4 26-31.
Takeuchi H, Kondo Y, Fujiwara K, Kanzawa T, Aoki H, Mills GB \& Kondo S 2005 Synergistic augmentation of rapamycin-induced autophagy in malignat glioma cells by phosphatidylnositol 3-kinase/protein kinase B inhibitors. Cancer Research 65 3336-3342.

Tam P \& Snow MHL 1981 Proliferation and migration of primordial germ cells during compensatory growth in mouse embryos. Journal of Embryology and Experimental Morphology 64 133-147.

Tilly JL 2001 Commuting the death sentence: how oocytes strive to survive. Nature 2 838-848.

Tilly JL \& Tilly KI 1995 Inhibitors of oxidative stress mimic the ability of follicle-stimulating hormone to suppress apoptosis in cultured rat ovarian follicles. Endocrinology 136 242-252.

Turner JMA, Mahadevaiah SK, Fernandez-Capetillo O, Nussenzweig A, Xu X, Deng C \& Burgoyne P 2004 Silencing of unsynapsed meiotic chromosomes in the mouse. Nature Genetics 37 41-47.

de Vries S, Baart EB, Dekker M, Siezen A, de Rooij DG, de Boer P \& de Riele H 1999 Mouse MutS-like protein Msh5 is required for proper chromosome synapsis in male and female meiosis. Genes and Development 13 523-531.

Wang R, Nakane PK \& Takeiko K 1998 Autonomous cell death of mouse germ cells during fetal and postnatal period. Biology of Reproduction $\mathbf{5 8}$ 1250-1256.

Wartenberg H, Ihmer A, Schwarz S, Miething A \& Viebahn C 2001 Mitotic arrest of female germ cells during prenatal oogenesis. A colcemid-like, non-apoptotic cell death. Anatomy and Embryology 204 421-435.

Yang DQ \& Kastan MB 2000 Partecipation of ATM in insulin signalling through phosphorylation of elF-4E-binding protein 1. Nature Cell Biology 2 893-898.

Yoshida K, Kondoh G, Matsuda Y, Habu T, Nishimune Y \& Morita T 1998 The mouse RecA-like gene Dmc1 is required for homologous chromosome synapsis during meiosis. Molecular Cell 1 707-718.

Received 26 March 2007

First decision 20 April 2007

Accepted 20 April 2007 